\title{
CRISPR-BASED GENE DRIVES FOR COMBATTING MALARIA: NEED FOR AN EARLY STAGE TECHNOLOGY ASSESSMENT
}

\author{
W. LIEBERT \\ Institute of Safety and Risk Sciences (ISR), \\ University of Natural Resources and Life Sciences (BOKU), Vienna, Austria; \\ liebert@boku.ac.at
}

\begin{abstract}
SUMMARY
The potential power of CRISPR-based gene drives makes it necessary to engage in science and technology assessment already in early stages of research and development. In order to argue for efforts to address this urgent need, gene drives to combat malaria-transmitting mosquitoes are discussed using the concept of prospective technology assessment. First, development risks are described, followed by considerations about anticipatable risks and irreversible consequences, as well as unforeseeable effects and uncertainties. Afterwards, fundamental problems in connection with the development of gene drives against malaria mosquitoes are raised. Opportunities for shaping technology are briefly discussed, before alternatives, in particular the World Health Organization's elimination strategy, are considered. Finally, several normative questions are put forward.
\end{abstract}

Key Words: Anticipation, gene drive, gene flow, malaria, malaria vector, mutagenic chain reaction, normative questions, prospective technology assessment, risk, selfish genetic element

\section{THE MALARIA CHALLENGE}

Malaria is caused by Plasmodium parasites which infected female mosquitoes can transmit to humans. Most of the malaria control strategies have been aimed at managing the vector of the malaria pathogen, i.e. the relevant mosquitoes of the genus Anopheles. However, not only the main malaria vector Anopheles gambiae Giles can transmit malaria, but in sum more than 30 Anopheles species (with highly varying regional distribution), i.e. roughly one percent of all known mosquito species can do so (NAS 2016).

J. Hendrichs, R. Pereira and M. J. B. Vreysen (eds.), Area-Wide Integrated Pest Management: Development and Field Application, pp. 795-808. CRC Press, Boca Raton, Florida, USA.

(C) 2021 IAEA 
The Plasmodium cycle first takes place in an infected mosquito (in particular inside the salivary glands) and after transmission in the liver and blood of a human. Some of the pathogens reach a sexual stage so that the whole malaria-parasite cycle (mosquito-human-mosquito) can start again after a bite of another mosquito which gets infected (White et al. 2014). Malaria is still endemic in many regions of the world. The World Health Organization (WHO) estimates that currently about 200 million humans are infected annually. In 2015, death cases due to malaria were in the range of 438000 , primarily in Africa (WHO 2015a).

The fight against malaria is regarded as one of the grand global challenges and was appropriately included in the UN Agenda for Sustainable Development in 2015. Control of the mosquito vector is mainly hampered by the development of resistance against the commonly used insecticides. Therefore, novel biotechnological strategies are under development and are coming into operation (for an overview cf. e.g. Alphey 2014). One such novel approach is a new tool of genetic engineering, i.e. the mutagenic chain reaction, mostly called gene drive, which comprises a variety of different technologies.

\section{GENE DRIVE AND PROSPECTIVE TECHNOLOGY ASSESSMENT}

Sexual reproduction provides, in principle, a $50 \%$ probability for the propagation of the parental genotypes to the progeny in accordance with Mendelian heredity rules. This evolutionary mechanism can be circumvented if specific parts of the genome can be transformed from heterozygote to homozygote, which has been observed in nature in some exceptional cases. Then, certain genotypes can be inherited with quite a high probability (significantly above $50 \%$ and up to $100 \%$ ) even if the fitness of the offspring is adversely affected.

In 2003, the British researcher Austin Burt proposed to genetically engineer such gene drives and to use them against malaria-transmitting mosquitoes (Burt 2003). The key idea was "to drive novel genes or mutations into wild populations" (Bull 2015). Theoretically, a rapid and targeted manipulation of entire mosquito populations would be possible while circumventing Mendelian rules. First attempts were made in the laboratory (e.g. Windbichler et al. 2011), but a research boom started only in 2013 with the discovery of the novel gene scissor CRISPR-Cas9 (Clustered Regularly Interspaced Short Palindromic Repeats-CRISPR-associated 9). The first CRISPRbased manipulation of the fruit fly Drosophila melanogaster Meigen in the laboratory was named as a mutagenic chain reaction by the researchers involved (Gantz and Bier 2015). Meanwhile, gene drives have been technically realized in the laboratory in yeast (DiCarlo et al. 2015) and in the malaria-transmitting mosquitoes Anopheles stephensi Liston (Gantz et al. 2015) and An. gambiae (Hammond et al. 2016).

Considering the malaria case in particular, gene drive approaches can be divided into suppression drives and modification/manipulation drives. Suppression drives aim at dramatically reducing or eradicating malaria-transmitting insect populations, regionally or globally. Modification/manipulation drives strive to genetically modify or manipulate mosquitoes in a way that malaria infection of humans is reduced or disabled. 
Gene drives could become extremely powerful tools for humans to make dramatic intentional or unintentional changes in populations and entire ecosystems. Therefore, it is necessary to engage in appropriate procedures of science and technology assessment in good time before such technologies are mature. An appropriate concept to guide such efforts is the approach of prospective technology assessment (ProTA) (Liebert and Schmidt 2010, 2015), which includes:

- analysis of scientific-technological development at an early stage, anticipating what might be relevant for science-based mid-term assessments and for (participatory) discourse inside and outside science

- assessment of intentions/visions, potentials, risks and unintended consequences, realistic potentials versus unrealistic visions and promises, uncertainties (and ignorance)

- characterization of the type of technology involved

- analysis/assessment of opportunities for shaping science and technology and of technical or socio-technical alternatives, so that desired potentials can be exploited

- reflection on normative issues, values and interests involved.

ProTA is more than an accompanying exercise of socio-economic research on societal or industrial acceptability, but it is partly impossible without analysing the scientific-technological core itself. ProTA differs also from outlining a development pathway for gene drive mosquitoes from research to use in the wild, including safe and efficient field-testing, regulations and post-implementation monitoring (James et al. 2018). In the following, above mentioned aspects of ProTA will be elaborated on. In doing so, sometimes more questions will be raised than answered.

\section{INTENTIONS, POTENTIAL AND DEVELOPMENT RISKS}

In general, research aiming to realize the potential of CRISPR-Cas9-based gene drives to reduce or eradicate malaria seems to be justified. The suffering of malaria victims worldwide, and in several African regions in particular, cannot be ignored by the international community. However, malaria is not only a naturally occurring phenomenon and a serious plague for humankind, but also an unpleasant result of human evolution that has provoked a long-lasting human and humanitarian struggle against it.

It is not only a question of scientific understanding and development of technical tools. Social, political and economic factors are also of great importance. Social organization and behaviour, access to modern healthcare, a functioning healthcare system in the regions concerned, preventive and curative measures and availability of suitable means are of utmost importance when trying to manage malaria. What we currently observe is an unjust societal and global divide with respect to the malaria burden, with prevention and medication affordable for the rich, but not for the poor. It is important to recognize that the remarkable social stratification of the malaria burden has almost nothing to do with new technological approaches to fight against malaria. 
Nevertheless, new biotechnical tools might provide helpful contributions to better manage malaria. Production and release of sterilized or Wolbachia-infected mosquitoes are already being validated in pilot projects to locally manage mosquito populations (Bourtzis et al. 2016). At first glance, CRISPR-based gene drives manipulating the genetic code of mosquitoes, at least theoretically, seem to be most promising in terms of efficacy. The release of one single modified organism could, in the medium-term, result in the modification of all individuals of a specific mosquito population (Noble et al. 2017). However, it turned out that in all of the abovementioned laboratory experiments the chain reactions are being reduced after a few generations. The engineered gene drives effectively became unstable (Gantz et al. 2015; Champer et al. 2016, 2017), which is not the case in naturally occurring selfish genes. Indeed, in the drive systems described so far, resistance alleles were detected after a few generations, highlighting a fundamental instability of engineered homingbased systems ${ }^{1}$. Thus, the potential of gene drives, which could be exploited in principle, has been made visible, but not more. Instead, development risks have emerged questioning whether the objective of a functional gene drive can be achieved. The observed slowdown of gene drives is partly due to the fact that, after a cleavage of targeted DNA due to CRISPR-Cas9, competing repair mechanisms are coming into play. With relevant probability non-homologous end joining (NHEJ) is sometimes faster and more prevalent than homology-directed repair (HDR), which only leads to the engineered homing endonuclease with self-replicating characteristics passing on the new genetic information from generation to generation. NHEJ can produce resistance against the drive and eventually stop the mutagenic chain reaction (Unckless et al. 2017; Champer et al. 2017).

It is not clear whether an evolutionary stable homing-based gene drive can be engineered (Bull 2015). Limited efficiency of gene drives could be regarded as something positive, in particular if one is afraid of a virtually unlimited efficacy of such engineered systems. However, it is possible to precisely predict the non-linear behaviour of gene drives in the wild? How exactly can mathematical modelling reproduce the actual complex dynamics? Can a persistent behaviour of a gene drive construct, which was originally predicted as eventually self-limiting, be excluded? It is being proposed to overcome resistance phenomena by combining several drives and targeting different DNA sites simultaneously. But that has not been demonstrated so far and it could turn out that complexity and instability would again be increased instead.

It is also questioned whether homologous recombination, which is essential for gene drives, is error-free (Guirouilh-Barbat et al. 2014). There are also doubts that gene drives in wild mosquito populations will be feasible. ${ }^{2}$ Although CRISPR-Cas9 is much easier, cheaper, faster, and more precise in usage than older tools of targeted genetic engineering, e.g. ZINC finger or TALEN (NAS 2016, p. 24-31; Häcker and Schetelig, this volume), off-target effects due to an incomplete specificity of constructed guide-RNAs cannot be excluded, which are associated with off-target DNA cleavages. These can contribute to an evolutionary instability of gene drives. Furthermore, cross-fertilization has been reported among various mosquito species (Tripet et al. 2011). Thus, one insect species could take the role of transmitting diseases from another. 
At the same time, it is also well known that Anopheles mosquitoes possess a large genetic diversity and evolutionary adaptability. In particular, that is the case for $A n$. gambiae, the primary carrier of Plasmodium in sub-Saharan Africa (The Anopheles gambiae 1000 Genomes Consortium 2017). Hence, the genetic variability of targeted mosquito species is a hurdle for an efficient gene drive strategy and a single gene drive might not be effective so that the development and use of many gene drive constructs might be needed to cover the range of genetic variants of Anopheles. Hence, functioning of mutagenic chain reactions or gene drives in malaria-transmitting mosquito populations is questioned from various sides. Development risks are numerous, but scientists eager to take on the challenges could ultimately overcome the obstacles. Therefore, one should be careful in stating that gene drives are not feasible; rather, one should anticipate that they can become a reality in the near future, and therefore there is an urgent need to study the risks and consequences involved.

\section{RISKS, CONSEQUENCES, AND UNCERTAINTIES}

What risks would be involved using gene drives to suppress insect pests in the wild? Unleashing a highly potent mutagenic chain reaction in insects like malariatransmitting mosquitoes could eventually spread across national borders. The population extinction programme could potentially work globally. This begs the following pertinent questions: who has the legitimate right to decide the initiation of such a mission and who will regulate it? Who will take part in the decision-making? What to do if approval is obtained in one country but not in the neighbouring country? Fundamentally, this would concern all living beings, and humankind as a whole should be asked. Could that be organized?

What, if modified mosquito genes mutate and further evolve, creating possibly unwanted variants? Could that be induced by off-target effects due to the fact that the specificity of guide RNAs targeting cleavage points in the DNA is not $100 \%$ ? Polymorphism as a genetic variation inside a population could be induced causing unclear consequences (Araki et al. 2014). Recently, a scientific debate has started over the importance of off-target effects. The creation of unintended single-nucleotide variants - not only small insertions and deletions (indels) - would fuel concerns. That can only be detected by whole-genome sequencing after gene-editing with CRISPRCas9. Unfortunately, that is seldom done by the researchers so far. ${ }^{3}$

Is it possible that after implementing the genetically engineered population suppression or modification against An. gambiae, Plasmodium falciparum Welch and/or other malaria parasites find a way to change their currently favoured or most important host mosquito? The ecological niche left open could be filled again. We already know about 30 mosquito species that can transmit malaria, but what would be the next move? Would this require efforts to eradicate more and more mosquito populations or species by engineering and unleashing more and more gene drives in nature? Moreover, the reduction of transmitting rates could lead to increased selection pressure on the pathogen itself, which could in turn develop an increased virulence (David et al. 2013). 
As cross-fertilization between different mosquito species has been observed (Fang 2010), an interspecific gene flow could affect other non-target mosquitoes or insects. This entails the potential of a gene drive-based eradication programme to jump over to other species (David et al. 2013), in particular if target sequences are equal. An effective elimination programme, originally targeting one species, could then result in significant consequences for ecosystems.

If targeted mosquito species play important roles in ecosystems or if other species are non-intentionally also affected, unwanted cascading effects in ecosystems are possible. Is it scientifically irrefutably clarified that malaria mosquitoes only have a damaging function in nature by infecting humans and other hosts? Do they have instead also important beneficial or indispensable roles in the food chain or in pollination processes? For mosquitoes in general, there are examples showing such vital purposes, like in the Camargue, in Nordic Arctic, or in aquatic systems (Fang 2010), but mainly we have to admit ignorance about side effects of eradicating malaria-transmitting Anopheles mosquitoes (David et al. 2013). However, a comparison in this respect with other methods of malaria vector control must not turn out negatively for gene drives.

Questions related to modification/manipulation drives indirectly aimed at the Plasmodium parasite or rendering the parasite harmless for humans are quite similar, even though the strategy would be different, i.e. not suppression or eradication. Offtarget effects could also have negative consequences and unwanted genetically engineered variants could emerge and reproduce. Accidentally, also the pathogenicity of the parasite could be increased.

It is well known that parasites evolve quickly and in unforeseen ways in relation to their hosts (Wijayawardena et al. 2013. A genetically engineered intervention into this interplay could result in unexpected or even damaging consequences. Could a mutating parasite like Plasmodium escape the grasp of newly engineered characteristics of the host mosquito? Would that just cheat the gene drive or probably even facilitate malaria transmission pathways or render the infections' impact on humans more likely or worse? Would that maybe provoke the engineering of another gene drive attempt and after the next one, another one, and so on?

Some scientists involved in gene drive research and development are warning that modified/manipulated drives are highly invasive in wild populations, even if their efficiency is low or resistance against the drive occurs (Noble et al. 2018). The question, among others, is what consequences will the spread of newly introduced alleles have? Is it possible to clarify this in advance? Is that also relevant for suppression drives, even if they are more or less limited in their potential to drive to fixation?

Thus, several risks and consequences of gene drives can be anticipated in all clarity. However, there are also plenty of uncertainties and unknowns due to the dynamic complexity of natural systems. Some important dynamical features are probably unknowable. A report of the US National Academy of Sciences (NAS 2016) has confirmed that many gaps exist in our knowledge on off-target effects of gene drives inside targeted organisms and on non-intended effects on other species and the environment. 


\section{FUNDAMENTAL PROBLEMS AND QUESTIONS RELATED TO GENE DRIVES}

If it is possible to engineer gene drives working effectively in a natural environment, then any single release of one gene drive modified organism could have irreversible consequences. The characteristics of the modified organism or the ramifications of the induced mutagenic chain reaction could also turn out as "wrong" or become detrimental, what is probably unknown prior to its release. Therefore, there is no tolerable limited release, as long as unwanted impacts cannot be completely excluded. The dual-use potential of gene drives seems to be obvious. Once it is sufficiently clarified that gene drives can be reliably engineered, small competent groups could (with only a limited amount of money) covertly pursue a strategy to engineer and use gene drives to the harm of others. One example could be an attacker (state or nonstate actor) who decides to manipulate an important organism that is beneficial for agriculture and that can only be found regionally or locally, with the aim to harm an adversary. Also, weapon-like effects of engineered gene drives are imaginable. ${ }^{4}$ Studying such possibilities in detail is therefore necessary prior to major investments in the development of gene drive technology.

With mutagenic chain reactions becoming a reality, the already significant depth of intervention by humans into natural processes would be massively increased. It has been proposed to differentiate genetic methods of mosquito control into more or less self-limiting approaches, and in increasingly self-sustaining invasive tools, which can or should persist in nature (Alphey 2014). Gene drives, in principle, correspond to the latter category and could become extremely powerful technologies, but with a high risk as unintended changes could become fully irreversible.

The precautionary principle, which is a guideline at least in the European context, would require an in-depth study of the risks of this new technology prior to any development in the laboratory and even more so prior to any consideration about application in nature. A number of serious risks and probable hazardous events due to gene drives have been already identified (cf. e.g. Hayes et al. 2018, p. S143). Those must be scrutinized and "Scientists must remain mindful that great power entails equally great responsibility, and take precautions accordingly" (Min et al. 2018, p. S54).

Unlike genetically modified organisms (GMOs), animals manipulated by gene drives are engineered with selfish genetic elements deliberately designed to spread in the environment and to operate autonomously in nature. Could these constructed or affected organisms evolve in a way not anticipated or even not anticipatable by their designers? Must we realize that a new form of technology is emerging?

Jan Schmidt $(2015,2016)$ has coined the term late-modern technology ("nachmoderne Technik") to identify a remarkable paradigm-shift. This new type of technology is based on the concept of self-organization and is linked to instabilities which can be triggered in non-linear dynamical systems. In contrast to the classicmodern type of technology, which is related to concepts of stability, linearization, predictability and controllability of functions and outcomes, in late-modern technology evolutionary, self-organizational, and non-linear features are exploited, which could lead in principle to intrinsic limits with respect to predictability and controllability. 
Organisms manipulated by an engineered gene drive are not only intentionally self-replicating but also capable of further evolutionary changes. This technology has to go through instabilities and has to trigger instabilities. It is a type of technology which acts nature-like in nature. However, this kind of built-in dynamic is provoking limitations with regard to the possibility of a stable construction of the technobiological system and also with regard to subsequent monitoring and control of the technology. A remarkable difference to classical modern and rational technology concepts is emerging.

Engineering gene drives to fight malaria could turn out as a harbinger of much more. Could gene drives against other mosquitoes, or against other insects in general or rodents transmitting infectious diseases be next? Gene drives could also be engineered against any so-called pest animals. Various tephritid fruit flies or plague locusts (Acrididae) could be a target. Also, non-native invasive species (plants as well as animals) could be attacked, eventually eradicated. All that is already mentioned in the NAS (2016) report. The research, development and use of gene drives against malaria-transmitting mosquitoes, which can be argued for based on convincing humanitarian objectives, could be the door-opener for a new dimension of human campaigns in and probably against nature or its biodiversity.

What starts with the fight against malaria could end up with nature being totally in the hands of humans ("Natur unter Menschenhand"), nature under complete management of humans, as the biologist and influential science manager Hubert Markl propagated already 20 years ago (Markl 1998, p. 147). Gene drives will dramatically change the way humans can interact with nature. Humans will have a tool then, to deliberately steer evolution - with less time for the ecosystems to adapt to the induced turbulence, in contrast to changes due to current tools and naturally occurring mutations. Humans can decide what species they like or dislike, which one has the right to survive in a given form, etc.

One of the young shooting-stars on the scientific scene (emerging from the group around George B. Church), Kevin Esvelt, already named his new working group at the Massachusetts Institute of Technology "Sculpting Evolution". Despite the farreaching visions, however, we are faced with huge unknowns of the complex, nonlinear interactions in genetic transfer, living cells and organisms, populations, ecosystems, and sensitive global life connections.

It appears that an intrinsic logic might underlie gene drive approaches against malaria-transmitting mosquitoes, as well as the other above-mentioned objectives: it is conceivable that after a first gene drive attempt more and more gene drives have to be engineered and released to correct or improve what wasn't achieved in the first step. As one gene drive will not suffice, the pressure for more will "naturally" be generated to bring about human control - which could turn out as being unachievable in the end. Do we have to expect a chain reaction of mutagenic chain reactions? A pathway towards life on earth totally in the hand of humans, prone to human errors and ignorance? 


\section{SHAPING OPPORTUNITIES FOR GENE DRIVES?}

Is there a chance to shape gene drives in a way that serious risks associated with them could be eliminated? Engineering "reversal drives" has been proposed that can undo results of a drive if necessary. But how to deal with off-target effects of both the original and the reversal drives? One is stuck in a principle problem. Therefore, even for several scientists involved in gene drive research, engineering reversal drives is not a convincing concept because a potential fundamental irreversibility of gene drives is admitted, and a reversal drive could only be a second drive which again could be ill-targeted.

Recently, self-limiting CRISPR-based drives have been proposed (Noble et al. 2019). But, so-called daisy-chain drives are just theoretical models and do not reflect on the real complex dynamics in real life. Furthermore, this concept seems to be more a test-bed approach, where drives at first (hopefully) are locally and timely restricted, but later a full-fleshed global release is intended. Furthermore, my impression is that it is more likely that all attempts to improve gene drives by additional features, proposed so far, increase the complexity and non-linearity of the engineered constructs and thus it is more likely that they would increase concerns.

\section{ALTERNATIVES TO GENE DRIVES}

If one hesitates to believe in gene drives as the new silver bullet against malaria, one has to refer to the alternatives. Several ideas for technical alternatives are currently discussed, researched, validated and are partly in use (Alphey 2014; Bourtzis et al. 2016; Fasulo et al., this volume; Häcker and Schetelig, this volume). One example is the infection of Aedes or Anopheles mosquitoes with Wolbachia bacteria (Incompatible Insect Technique or IIT), which are maternally inherited and affect reproduction capabilities, leading to strategies to suppress or replace mosquito populations (Bourtzis et al. 2016). Another example of population suppression is the release of genetically modified mosquitoes passing on dominant lethal factors to their offspring (RIDL) (Alphey 2014; but see Evans et al. (2019).

Another approach, which has been in use for over 50 years on all continents against major agricultural pest insects, is the Sterile Insect Technique (SIT), where large numbers of the target insects are mass-reared, sterilized by radiation, and then released in order to negatively influence the reproduction of insect populations (Dyck et al. 2021).

Multiple releases of sufficient quantities of manipulated mosquitoes are necessary in all these cases (when population suppression rather than replacement is the objective), to obtain the wanted results. Such approaches can also be debated and must be carefully assessed. Obeying the precautionary principle is also mandatory for these technologies. But, in principle, one could say, that unpleasant risks and other ramifications of these alternative technologies might be less severe than in the case of gene drives. In the case of the SIT, there are decades of track record of successful large-scale application against many pest insects. As the released insects are sterile, they cannot become established, and thus there is no irreversibility. ${ }^{5}$ 


\section{GLOBAL PROGRAMME TO ELIMINATE MALARIA}

Of particular importance with regard to alternatives is the global programme to eliminate malaria. The United Nations and the WHO have declared the intent of reaching this goal by 2030 (WHO 2015b) using the following classical methods:

- vector control, in particular by distribution of long-lasting insecticidal bed nets; indoor residual spraying; mosquito screening, surveillance and monitoring; and education of citizens in endemic regions

- prevention, in particular preventive intermittent chemoprophylactic treatment of pregnant women and children under 5 years, especially in many African regions

- better access and use of diagnostic testing and appropriate medical treatment (e.g. artemisinin-based combination therapy).

In this century, important successes in the fight against malaria have already been recorded: a reduction of malaria incidence by $37 \%$ and of mortality rate by $60 \%$ (WHO 2015a). Therefore, the hopes are high that the malaria elimination strategy can be accomplished, if sufficient funding can be raised (much more than USD 2.5 billion annually, which was the global financing for malaria control in 2014, will be needed) and if a concerted effort and enduring engagement of all stakeholders can be achieved over the next coming years. In this struggle, unglamorous tasks such as improvement or set up a minimally functional health care system are critical, including durable and affordable access to diagnostics and pharmaceuticals, as well as educating and empowering communities so that they can reduce the risk themselves. A success with this strategy would also have further positive ramifications not just with respect to malaria.

It is clear that the global programme to eliminate malaria involves much more than just high technological means such as gene drives. The malaria challenge is not only a problem solvable with scientific-technological approaches, but also social, political and economic factors have to be addressed. Not only is vector control crucial, but in the end, control of $P$. falciparum and other parasites is of utmost importance. The parasite cycle has to be interrupted in a sustainable manner, which is much more an issue of health care, access to suitable simple measures, societal development, etc. than using sophisticated novel technologies. Without a somewhat stable (minimal) health care system in the endemic regions of concern, elimination of malaria is impossible. Also, the socio-economic conditions of the disease's origin, besides the natural-scientific causes for malaria, need to be scrutinized in order to find appropriate means to support the transition process towards malaria elimination. Population growth of mosquitoes has also to do with socio-cultural or techno-economic change, for example the rapid increase of plastic containers used for food distribution or scrap tyres provide some mosquitoes with ideal breeding grounds.

One should also remark that in several countries past (successful) elimination campaigns also had harmful side effects on the environment by massive use of DDT; furthermore, first resistances of mosquitoes against insecticides developed at that time. Improper use of malaria drugs, which mostly have to be taken in suitable combinations, has also led to resistance of the malaria parasite. 


\section{NORMATIVE QUESTIONS}

Many issues and values involved in the necessary debate on gene drives are mentioned in the NAS (2016) study "Gene drives on the horizon". Many scientists state: "risk has to be balanced against benefit", which can lead to a purely utilitarian position. Clearly, weighing positive and negative consequences is a relevant part, but ethical reflection should heed all ramifications and also fundamental problematics.

Derived from Hans Jonas' principle of responsibility (Jonas 1979), which aims at achieving a "conservative" preservation of our lifeworld, the precautionary principle focuses mainly on objective reasoning in respect to serious risks, notwithstanding that benefits might also be possible. On the other hand, an unfolding principle, which strives for "alliance technology" serving humankind and being concurrently in harmony with nature (Bloch 1959), requests a positive and socially just developmental progression of humankind by using new technology that is bound to its alliance with nature, in harmony with nature. Both principles which may be contradictory at a first glance can be used as normative orientation ${ }^{6}$, already in the process of research and development, when striving for the eradication of malaria (probably using gene drives).

The perceived role of humans in nature is highly relevant: what position has humankind within nature, still being a central part of our common lifeworld which we share with other living beings? One position claims a human role as the manager of all life on earth, man as "master of nature" (Descartes), the other sees humankind embedded into nature and as partner of life on earth, or as Albert Schweitzer has formulated it in his principle respect for life (Schweitzer 1966): I am life that wants to live in the middle of life that wants to live.

How should members of the scientific community and of our societies behave in between these diametrically opposite positions? Schweitzer's position seems to be incompatible with an approach where humankind feels entitled to steer evolution on earth. Who is entitled to change nature in a way that it could irreversibly affect all life on earth? A single researcher, the scientific community, a competent national agency, a nation?

In any case, the whole fabric of risks, uncertainties and ignorance, and the possible dramatic consequences of mutagenic chain reactions, will (and must) have a massive influence on ethical discourse debating responsible conduct in gene drive $R \& D$ directed towards practical use, and other technology-based malaria elimination strategies. In the end, it has to be assessed which procedure for malaria elimination seems to be promising, associated with low risk, and is globally, societally and ethically acceptable. I deem it as obvious that the WHO strategy cannot be replaced by anything else. But maybe additional new measures or tools could be helpful.

All potentials, risks, uncertainties, ramifications of gene drive R\&D have to be made transparent within science and to the broader public as early as possible. When a gene drive technology against malaria-transmitting mosquitoes seems to be mature - and that could soon be the intuition of several researchers and funding organizations (like the Gates Foundation) involved - it would probably be too late to be withheld. Then it will be no longer possible to stop its use in a region with serious malaria burden. 
The hope of promoters is that some advantages will predominate notwithstanding those arguments describing possible or anticipatable negative side effects or long-term consequences, serious uncertainties and ramifications that have been put forward. All the concerns will then be covered-up by promises and hopes.

In conclusion, using the example of combatting malaria, this contribution tried to substantiate why prospective technology assessment in the field of gene drive research and development is urgent and what issues should be assessed in order to provide input for decision making inside and outside science. The pertinent questions to be answered are going beyond tailored disciplinary research fields of scientific specialty. As scientists involved in gene drive research have put it:

"Determining whether, when, and how to develop gene drive interventions responsibly will be a defining challenge of our time" (Min et al. 2018, p. S40).

\section{NOTES}

(1) More precisely: homing endonuclease genes (HEGs) or homing-based gene drives.

(2) The entomologist Flaminia Catteruccia is cited with: “...you can have the fanciest technology on earth, the perfect gene drive, but if your laboratory mosquitoes can't mate with wild mosquitoes, then it's not going to work at all" (Shaw 2016).

(3) Schaefer et al. (2017) had reported in Nature an unexpected high number of unintended single-nucleotide variants after genome editing with CRISPR-Cas9 in a laboratory population of mice. After several criticisms, the Nature editors have withdrawn the paper because it could not be shown beyond any doubt that the use of the gene scissor was the cause of these effects. However, they admitted that Schaefer et al. "did not examine only predicted sites", as in many other studies, "but looked at the entire genome" and that the "work of Schaefer et al. highlights limitations in the current literature that should be considered" (Nature Methods 15 (4): 229-230).

(4) At end of 2017, it has been revealed that the US Defense Advanced Research Projects Agency (DARPA) is investing 100 million USD in gene drive technology (http://genedrivefiles.synbiowatch.org/).

(5) Therefore, sterile insects are accepted as beneficial organisms by the International Plant Protection Convention (FAO 2005), to which 183 countries are signatories.

(6) More about these viewpoints can be found in Liebert and Schmidt (2015).

\section{REFERENCES}

Alphey, L. 2014. Genetic control of mosquitoes. Annual Review of Entomology 59: 2015-224.

Araki, M., K. Nojima, and T. Ishii. 2014. Caution required for handling genome editing technology. Trends in Biotechnology 52 (5): 234-237.

Bourtzis, K., R. S. Lees, J. Hendrichs, and M. J. B. Vreysen. 2016. More than one rabbit out of the hat: Radiation, transgenic and symbiont-based approaches for sustainable management of mosquito and tsetse fly populations. Acta Tropica 157: 115-130.

Bloch, E. 1959. Prinzip Hoffnung. Suhrkamp-Verlag, Frankfurt, Germany. English translation: The principle of hope. Cambridge, Massachusetts, USA, 1986.

Bull, J. 2015. Evolutionary decay and the prospects for long-term disease intervention using engineered insect vectors. Evolution, Medicine, and Public Health: 152-166.

Burt, A. 2003. Site-specific selfish genes as tools for the control and genetic engineering of natural populations. Proceedings of the Royal Society B: Biological Sciences 270: 921-928.

Champer, J., A. Buchman, and O. Akbari. 2016. Cheating evolution: Engineering gene drives to manipulate the fate of wild populations. Nature 17: 146-159. 
Champer, J., R. Reeves, S. Y. Oh, C. Liu, J. Liu, A. G. Clark, and P. W. Messer. 2017. Novel CRISPR/Cas9 gene drive constructs reveal insights into mechanisms of resistance allele formation and drive efficiency in genetically diverse populations. PLoS Genetics 13 (7): e1006796.

David, A., J. Kaser, A. Morey, A. Roth, and D. Andow. 2013. Release of genetically engineered insects: A framework to identify potential ecological effects. Ecology and Evolution 3: 4000-4015.

DiCarlo, J., A. Chavez, S. Dietz, K. Esvelt, and G. Church. 2015. Safeguarding CRISPR-Cas9 gene drives in yeast. Nature Biotechnology 33: 1250-1255.

Dyck, V. A., J. Hendrichs, A. S. Robinson (eds.). 2021. Sterile Insect Technique - Principles and practice in Area-Wide Integrated Pest Management. Second Edition. CRC Press, Boca Raton, Florida, USA. 1200.

Evans, B. R., P. Kotsakiozi, A. L. Costa-da-Silva, R. S. Ioshino, L. Garziera, M. C. Pedrosa, A. Malavasi, J. F. Virginio, M. L. Capurro, and J. R. Powell. 2019. Transgenic Aedes aegypti mosquitoes transfer genes into a natural population. Scientific Reports 9: 13047.

Fang, J. 2010. A world without mosquitoes. Nature 466: 432-434.

(FAO) Food and Agriculture Organization of the United Nations. 2005. International Standard for Phytosanitary Measures (ISPM) No. 3, Guidelines for the export, shipment, import and release of biological control agents and other beneficial organisms. FAO, Rome, Italy.

Gantz, V., and E. Bier. 2015. The mutagenic chain reaction: A method for converting heterozygous to homozygous mutations. Science 348: 442-444.

Gantz, V., N. Jasinskiene, O. Tatarenkova, A. Fazekas, V. Macias, E. Bier, and A. James. 2015. Highly efficient Cas9-mediated gene drive for population modification of the malaria vector mosquito Anopheles stephensi. Proceedings of the National Academy of Sciences 112: 6736-6743.

Guirouilh-Barbat, J., S. Lambert, P. Bertrand, and B. Lopez. 2014. Is homologous recombination really an error-free process? Frontiers in Genetics 5: 175.

Hammond, A., R. Galizi, K. Kyrou, A. Simoni, C. Siniscalchi, D. Katsanos, M. Gribble, D. Baker, E. Marois, S. Russell, A. Burt, N. Windbichler, A. Crisanti, and T. Nolan. 2016. A CRISPR-Cas9 gene drive system targeting female reproduction in the malaria mosquito vector Anopheles gambiae. Nature Biotechnology 34: 78-83.

Hayes, K., G. Hosack, G. Dana, S. Foster, J. Ford, R. Thresher, A. Ickowicz, D. Peel, M. Tizard, P. De Barro, T. Strive, and J. Dambacher. 2018. Identifying and detecting potentially adverse ecological outcomes associated with the release of gene-drive modified organisms. Journal of Responsible Innovation 5(S1): S139-S158.

James, S., F. H. Collins, P. A. Welkhoff, C. Emerson, H. C. J. Godfray, M. Gottlieb, B. Greenwood, S. W. Lindsay, C. M. Mbogo, F. O. Okumu, H. Quemada, M. Savadogo, J. A. Singh, K. H. Tountas, and Y. T. Toure. 2018. Pathway to deployment of gene drive mosquitoes as a potential biocontrol tool for elimination of malaria in Sub-Saharan Africa: Recommendations of a scientific working group. American Journal of Tropical Medicine and Hygiene 98 (Supplement 6): 1-49.

Jonas, H. 1979. Das Prinzip Verantwortung. Suhrkamp-Verlag, Frankfurt, Germany. English translation: The imperative of responsibility: In Search for an ethics of the technological age. Chicago, USA, 1984.

Liebert, W., and J. Schmidt. 2010. Towards a prospective technology assessment: Challenges and requirements for technology assessment in the age of technoscience. Poiesis \& Praxis 7: 99-116.

Liebert, W., and J. Schmidt. 2015. Demands and challenges of a prospective technology assessment, pp. 331-340. In C. Scherz, T. Michalek, L. Hennen, L. Hebáková, J. Hahn, and S. Seitz (eds.), The next horizon of technology assessment. Technology Centre ASCR, Prague, Czech Republic.

Markl, H. 1998. Wissenschaft gegen Zukunftsangst. Hanser-Verlag, München/Wien, Germany/Austria.

Min, J., A. Smidler, D. Najjar, and K. Esvelt. 2018. Harnessing gene drive. Journal of Responsible Innovation 5(S1): S40-S65.

(NAS) National Academies of Sciences. 2016. Gene drives on the horizon: Advancing science, navigating uncertainty, and aligning research with public values. The National Academies Press. Washington, DC, USA.

Noble, C., J. Olejarz, K. Esvelt, G. Church, and M. Nowak. 2017. Evolutionary dynamics of CRISPR gene drives. Science Advances 3(4): e1601964

Noble, C., B. Adlam, G. Church, K. Esvelt, and M. Nowak. 2018. Current CRISPR gene drive systems are likely to be highly invasive in wild populations. eLife 7: e33423

Noble, C., J. Min, J. Olejarz, J. Buchtahl, A. Chavez, A. Smidler, E. DeBenedictis, G. Church, M. Nowak, and K. Esvelt. 2019. Daisy-chain gene drives for the alteration of local populations. Proceedings of the National Academy of Sciences 116: 8275-8282. 
Schaefer, K., W.-H. Wu, D. Colgan, S. Tsang, A. Bassuk, and V. Mahajan. 2017. Unexpected mutations after CRISPR-Cas9 editing in vivo. Nature Methods 14 (6): 547-548.

Schmidt, J. C. 2015. Synthetic biology as late-modern technology, pp. 1-30. In B. Giese, C. Pade, H. Wigger, and A. von Gleich (eds.), Synthetic biology, character and impact. Springer-Verlag, Cham/ Heidelberg, Switzerland/Germany.

Schmidt, J. C. 2016. Philosophy of late-modern technology, pp. 13-29. In J. Boldt (ed.), Synthetic biology, metaphors, worldviews, ethics, and law. Springer-Verlag, Cham/Heidelberg, Switzerland/ Germany.

Schweitzer, A. 1966. Die Ehrfurcht vor dem Leben. Grundtexte aus fünf Jahrzehnten. Verlag C. H.Beck, Nördlingen, Germany. 167 pp.

Shaw, J. 2016. Editing an end to malaria? The promise, and possible perils, of a new genetic tool. Harvard Magazine, May-June 2016.

The Anopheles gambiae 1000 Genomes Consortium. 2017. Genetic diversity of the African malaria vector Anopheles gambiae. Nature 552: 96-100.

Tripet, F., P. Lounibos, D. Robbins, J. Moran, N. Nishimura, and E. Blosser. 2011. Competitive reduction by satyrization? Evidence for interspecific mating in nature and asymmetric reproductive competition between invasive mosquito vectors. American Journal of Tropical Medicine and Hygiene 85: 265-270.

Unckless, R., A. Clark, and P. Messer. 2017. Evolution of resistance against CRISPR-Cas9 gene drive. Genetics 205: 827-841.

White, N., S. Pukrittayakamee, T. Hien, A. Faiz, O. Mokuolu, and A. Dondorp. 2014. Malaria. The Lancet 383: 723-735.

(WHO) World Health Organization. 2015a. World malaria report 2015. WHO Press, Geneva, Switzerland.

(WHO) World Health Organization. 2015b. Global technical strategy for malaria 2016-2030. WHO Press, Geneva, Switzerland.

Wijayawardena, B., D. Minchella, and J. DeWoody. 2013. Hosts, parasites and horizontal gene transfer. Trends in Parasitology 29: 329-338.

Windbichler, N, M. Menichelli, P. Papathanos, S. Thyme, H. Li, U. Ulge, B. Hovde, D. Baker, R. Monnat, A. Burt, and A. Crisanti. 2011. A synthetic homing endonuclease-based gene drive system in the human malaria mosquito. Nature 473: 212-217. 